\title{
RUGOSIDAD Y TEXTURA DE SUPERFICIES: EXPERIMENTOS Y SIMULACIONES
}

\section{SURFACE ROUGHNESS AND TEXTURE: EXPERIMENTS AND SIMULATIONS}

Fecha de Recepción: 30 de Agosto de 2006 Fecha de Aprobación: 11 de Octubre de 2006

\author{
Watson L. Vargas ${ }^{1-2}$ \\ Lyda M. Pineda ${ }^{3}$ \\ Luz Elena Santaella ${ }^{4}$
}

RESUMEN: En este trabajo se aplican algunos elementos de la geometría fractal al estudio y caracterización de la rugosidad superficial. Los materiales bajo estudio consisten en agregados naturales, de amplia aplicación en la industria de la construcción. Se aplican tanto técnicas experimentales basadas en análisis de imágenes como modelos teóricos. En particular se explora el potencial de la lacunaridad -un método de análisis derivado de la geometría fractal, para describir patrones y dispersión espacial. Los resultados ilustran su aplicación en la caracterización de rugosidad superficial. Se discuten igualmente, limitaciones y ventajas de esta aproximación.

PALABRA CLAVES: Geometría fractal, rugosidad, lacunaridad, dimensión fractal.

ABSTRACT: In this work, some elements of fractal geometry are used to study and characterize surface roughness. The materials under study are natural aggregates commonly used in the construction industry. Both experimental and theoretical approaches are used. In particular, we explore lacunarity analysis - a multi-scaled method derived from fractal geometry- used for the describing patterns and dispersion. We illustrate its application to characterize surface roughness. Limitations and advantages of this approach are also discussed.

KEYWORDS: Fractal geometry, roughness, lacunarity, fractal dimension.

1 Profesor Asociado, Facultad de Ingeniería (UMNG) Ing. Químico, MSc., Ph.D Autor corresponsal: wvargas@etb.net.co

2 Dirección actual: 1249 Benedum Hall, Department of Chemical and Petroleum Engineering University of Pittsburgh, Pittsburgh, PA 15261

3 Ing. Civil, Joven Investigadora programa UMNG

4 Profesora asistente, Facultad de Ingeniería (UMNG), Ing. Civil, Ph.D 


\section{INTRODUCCIÓN}

El análisis de superficies rugosas tiene aplicaciones obvias y algunas otras más subjetivas en diversos campos de la ingeniería. Por ejemplo, una rugosidad superficial de tan solo unos cuantos nanómetros es suficiente para remover la adherencia entre dos superficies sólidas elásticas[ ], aún la ablación de superficies glaciares sigue comportamientos que generan superficies rugosas denominadas penitentes de importancia significativa en el estudio de procesos de erosión terrestre y de seguimiento de cambio climático[ ]. La formulación de modelos y técnicas que puedan caracterizar la topografía de superficies rugosas ha sido un área de investigación activa por muchas décadas. Por casi un siglo la comunidad científica ha lidiado con el problema de caracterizar la geometría de superficies, usando una gran variedad de modelos matemáticos y técnicas de análisis[ ]. Dichos trabajos han mostrado resultados notables, sin embargo, existen aún descripciones incompletas. La historia de esta área de investigación inicio fundamentalmente con descripciones estadísticas, con un giro reciente hacia investigaciones basadas en geometría fractal. Aun cuando la investigación acumulada sobre superficies rugosas nos ha suministrado conocimiento considerable, existen problemas fundamentales aún por resolver.

Los esfuerzos iniciales encaminados a desarrollar una caracterización rigurosa de las superficies rugosas de aplicación en ingeniería, son trabajos esencialmente estadísticos cuyo objetivo primario es el de describir la distribución de asperezas, basándose fundamentalmente en la construcción de una distribución Gaussiana i.e., aleatoria, de las elevaciones de la superficie. Estas aproximaciones iniciales funcionan bien para un buen número de aplicaciones de ingeniería. Como resultado de esto, más de 30 parámetros diferentes han sido adoptados para cuantificar la topografía superficial. En consecuencia, parámetros estadísticos de la distribución de alturas superficiales tales como el rms $\sigma$ (Root Mean Square), por sus siglas en ingles; la pendiente, $\sigma^{\prime}$ y la curvatura $\sigma^{\prime \prime}$, son usados con frecuencia para caracterizar superficies y para modelar contactos elasto-plasticos de cuerpos sólidos isotropicos y anisotropicos.

En años recientes sin embargo, se han identificado serias deficiencias en estos modelos, las cuales han sido ampliamente discutidas en la literatura. Entre las más destacadas, están: la distribución no estacionaria de las alturas, las características multi-escalares de la geometría de la superficie y tal vez lo más complicado, es el hecho de que la medida estadística de la rugosidad superficial tiende a ser dependiente de la resolución del instrumento utilizado, el tamaño de la muestra y de filtros experimentales utilizados para adquirir los datos topográficos. A pesar de estos inconvenientes, las medidas estadísticas continúan aportando conocimiento fundamental sobre la naturaleza de la rugosidad.

Las limitaciones descritas arriba, junto con los perfiles auto-similares, han sugerido que la geometría fractal puede ser una mejor forma de describir la rugosidad superficial. Esta aproximación novedosa fue originalmente propuesta por Mandelbrot[4] y desde entonces ha recibido gran atención por parte de la comunidad científica $[5,6,7,8]$. Por ejemplo, en el caso de las superficies rugosas fractales, $\sigma^{\prime}$ y $\sigma^{\prime \prime}$, dependen de la resolución del instrumento utilizado, pero no del tamaño de la muestra, mientras que $\sigma$, depende del tamaño de la muestra y es insensible a la resolución del instrumento [6]. Muchas superficies de interés en ingeniería, presentan características propias de los fractales sobre un rango amplio de longitudes, esta característica ilustra el potencial de la geometría fractal para representar textura superficial desde la micro-escala hasta la nano-escala. Los modelos fractales han sido aplicados con éxito a la descripción cualitativa y cuantitativa de rugosidad superficial, caracterización de forma y textura, caracterización de superficies. 
El objetivo primario de esta publicación, es por lo tanto, introducir algunos conceptos de geometría fractal aplicados a la caracterización de superficies rugosas y presentar resultados experimentales y numéricos de la representación de superficies rugosas aplicando herramientas de la geometría fractal. El artículo se organiza como sigue: la Sección II presenta algunos conceptos fundamentales sobre la caracterización fractal de superficies. También se realiza una comparación cualitativa entre los modelos matemáticos y algunas observaciones experimental. En la Sección III se describen los métodos experimentales y de procesamiento de imágenes aplicados en el presente estudio. En la Sección IV se presentan resultados sobre la caracterización de rugosidad superficial, aplicando dos parámetros derivados de la geometría fractal i.e., dimensión fractal y lacunaridad. Finalmente en la Sección IV se presentan algunas conclusiones y perspectivas.

\section{CARACTERIZACIÓN FRACTAL DE SUPERFICIES}

La geometría fractal, introducida por Mandelbrot [2] puede observase en varios fenómenos naturales, dentro de los que se incluyen, turbulencia, precipitaciones, terremotos, la topografía de superficies y se caracteriza primordialmente por su continuidad, no-diferenciabilidad y auto-afinidad. Estas características propias de los fractales hacen que en general su geometría se describa no por una ecuación tal como $y=f(x)$, sino mediante relaciones recurrentes, e.g., $x_{i+2}=f\left(x_{i+1}\right)=f\left(f\left(x_{i}\right)\right)$. La función de movimiento Browniano fraccional, es una función derivada de la geometría fractal que cuando se extiende de una curva a una superficie -con una dimensión fractal dada - simula una topografía superficial. La función de Weierstrass-Mandelbrot está dada por

$$
w(x)=\sum_{n=-\infty}^{\infty} y^{\left(D_{s}-2\right) n}\left(1-e^{i y^{n} x}\right) e^{i \phi_{n}}
$$

donde, $w$ es una función compleja de variable real $x$. En la Ecuación $1,1<D_{s}<2$, es la dimensión fractal del perfil, $n$ es el índice de frecuencias, $\phi_{n}$ es una fase aleatoria, y $y>1$ es un parámetro que determina la densidad de las frecuencias en el perfil. y representa físicamente la resolución espacial o lacunaridad del perfil. Un perfil fractal $z(x)$ que semeja el perfil topográfico de una superficie, se puede obtener al tomar la componente real de $w(x)$,

$$
z(x)=\operatorname{Re}[w(x)]=\sum_{n=-\infty}^{\infty} \gamma^{\left(D_{x}-2\right) x}\left[\cos \phi_{n}-\cos \left(\gamma^{n} x+\phi_{n)}\right]\right.
$$

Un ejemplo de perfiles generados aplicando la Ecuación 2 se ilustran en la Figura 1.

La función de Weierstrass-Mandelbrot es básicamente una superposición infinita de funciones coseno a frecuencias discretas y con una fase aleatoria, con un cierto espaciamiento entre frecuencias sucesivas (la lacunaridad), y un exponente $D_{s}$ que escala la amplitud por la frecuencia. $D_{s}$ está relacionado con el exponente de Hurst -un parámetro de medida común en fractales--, mediante la relación $D_{s}=2-H$, para perfiles en dos dimensiones. Para propósitos de implementación práctica, generalmente la lacunaridad se toma aproximadamente igual a 1.5 y la sumatoria se efectúa sobre un rango relativamente bajo, e.g. $n=3-8$, octavas (octavas $\alpha$ duplicación de las frecuencias). Sumatorias más grandes son innecesarias y en algunos casos pueden ser perjudiciales para el resultado buscado. El espectro de potencia $P(\omega)$, del perfil descrito por la Ecuación 2, está dado por

$$
P(\omega)=\frac{1}{\omega^{(5-2 D s)} \ln \gamma}
$$

una función inversa de la frecuencia espacial [6], , dicho espectro se ha demostrado experimentalmente, es aplicable a muchas superficies de interés en ingeniería. 

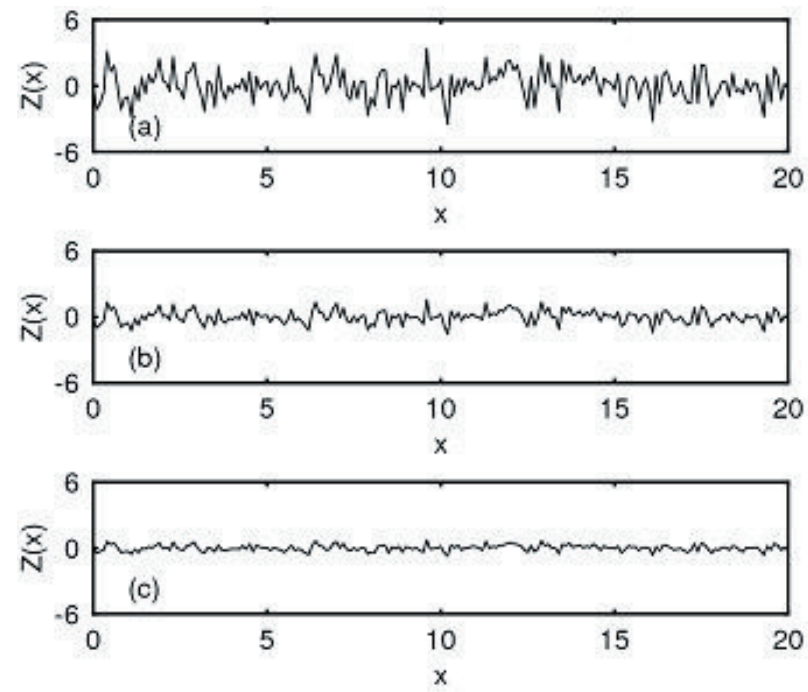

Figura 1. Perfiles de rugosidad simulados aplicando la Ecuación 2. (a) $H=0.8$, (b) $H=0.5$, (c) $H=0.2$.

Ausloos y Berman [9] generalizaron la función de Weierstrass-Mandelbrot en la Ecuación 1, mediante la introducción de múltiples variables que tienen en cuenta procesos estocásticos de más alta dimensionalidad. La función en dos variables propuesta por Ausloos y Berman puede usarse para modelar superficies fractales con corrugaciones en todas las direcciones. La función de altura de las asperezas que muestra aleatoriedad en todas las direcciones, corresponde a la parte real de la función de AusloosBerman y está dada por

$$
\begin{aligned}
z(x, y)= & L\left(\frac{G}{L}\right)^{\left(D_{z}-2\right)}\left(\frac{\ln \gamma}{M}\right)^{1 / 2} \sum_{m=1}^{M} \sum_{n=0}^{n_{m a n}} \gamma^{\left(D_{x}-3\right) n} \\
& \times\left\{\cos \phi_{m, n}-\cos \left[\frac{2 \pi \gamma^{n}\left(x^{2}+y^{2}\right)^{1 / 2}}{L} \times \cos \left(\tan ^{-1} \frac{y}{x}-\frac{m n}{M}\right)+\phi_{m, n}\right]\right\}
\end{aligned}
$$

En la Ecuación 4, tiene el mismo significado y magnitud que en la Ecuación 2 y $2<D_{s}<3$, es la dimensión fractal de la superficie. En la Ecuación 4, G es un parámetro de escalamiento de la altura promedio de las asperezas, independiente de la frecuencia y que se ha denominado la rugosidad fractal. El significado físico de $D_{s}$ es el de cuantificar la extensión del espacio cubierto por la superficie fractal, a valores altos de $D_{s}$ corresponden perfiles más densos. Para superficies isotropicas, $D_{s}$ puede determinarse de la pendiente de un grafico log-log del espectro de potencia, igualmente puede obtenerse mediante técnicas de análisis de imagen aplicando software especializado para tal fin. Esta última aproximación será la que se utilice en el presente estudio. El parámetro $M$ denota el número de picos superpuestos, usados para construir la superficie. $\phi_{m, n}$, es la fase aleatoria, para lo cual se utiliza un generador de números aleatorios que distribuye los valores en el intervalo [0,2 $\pi$ ]. Para propósitos prácticos, el índice $n$, asume valores finitos. Dado que la frecuencia más baja es igual a $1 / L$, donde $L$ es la longitud de la muestra, el límite inferior de $n$ puede fijarse en cero. Sin embargo para determinar la fuerza de contacto, aplicando los diversos modelos constitutivos existentes, la longitud de onda más baja no puede ser menor a una longitud mínima $L_{s}$. La frecuencia más alta es igual a $1 / L_{s}$, y el límite superior de $n$ está dado por

$$
n_{\operatorname{mxx}}=\operatorname{int}\left[\frac{\log \left(L / L_{s}\right)}{\log \gamma}\right]
$$

donde int[...] denota el máximo valor entero del número en paréntesis. La Ecuación 4 ha sido utilizada extensamente para representar superficies fractales isotropicas [6, 7]. La Figura 2 ilustra el resultado físico de $D_{s}$ sobre la topografía de una superficie. Las simulaciones se efectuaron aplicando una rutina computacional desarrollada en Matlab ${ }^{\circledR}$. La comparación de estas dos topografías indica que, para una rugosidad fractal $G$ y parámetro $M$ constantes, un incremento en la magnitud de $D_{s}$ genera superficies de topografía más suave. 


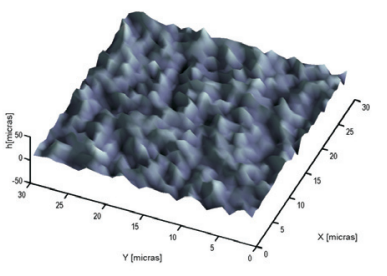

(a)

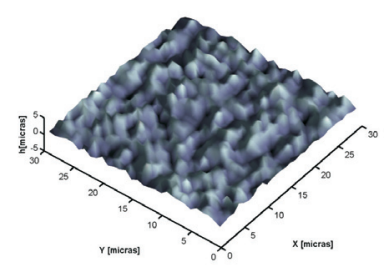

(b)
Figura 2. Superficies fractales simuladas aplicando la Ecuación 4. (a) $D_{s}=2.4$, (b) $D_{s}=2.8$. $, G=1.36 e-2, M=10, L=30 \mu \mathrm{m}$

Como una demostración del potencial de estas técnicas, en la Figura 3 consideramos un ejemplo comparativo de las superficies fractales naturales $3(a)$, simuladas bajo condiciones de laboratorio $3(\mathrm{~b})$ y simuladas matemáticamente (3c), aplicando la función de Ausloos-Berman (Ec. 4). El modelo prototipo corresponde a los denominados penitentes formaciones glaciares características de la alta montaña de la cordillera de los andes. Dichas formaciones se generan mediante procesos de ablación causada por la radiación solar directa y reflejada [2]. Nótese que el modelo matemático reproduce cualitativamente de manera muy cercana la topografía tanto natural como la simulada bajo condiciones de laboratorio y por lo tanto puede servir como complemento para entender mejor los procesos que llevan a la formación de tales estructuras

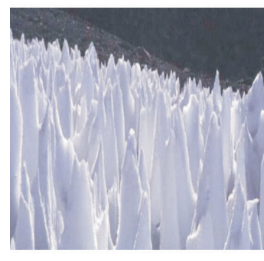

(a)

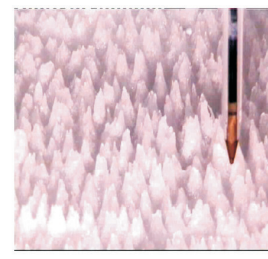

(b)

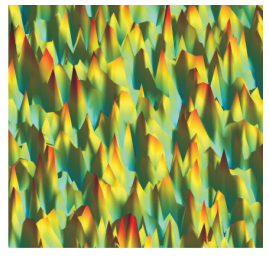

(c)
Figura 3. Superficies fractales. (a) Penitentes naturales en la cordillera de los Andes. (b) modelo experimental de ablación de un glaciar, bajo condiciones de laboratorio. Imagen tomada de la información complementaria Ref. [2]. (c) Superficie fractal simulada usando la Ecuación 4, aplicando ruido de tipo Levy para la fase aleatoria $\phi_{m, n}$.

\section{MÉTODO EXPERIMENTAL Y PROCESAMIENTO DE IMÁGENES}

La rugosidad resulta del proceso de manufactura particular de un producto o de la condición natural de un material. En este trabajo se estudia la rugosidad de agregados naturales, dado que la rugosidad superficial de estos materiales es muy importante en diversas aplicaciones de la industria de la construcción. El material seleccionado para estudio corresponde a agregados de origen aluvial, extraídos de canteras de la sabana de Bogotá. Materiales con una amplia variación visual de la "rugosidad" fueron seleccionados en el sitio de colección y posteriormente agrupados en muestras de cinco grupos distintos de acuerdo con la "rugosidad" visual observada. La rugosidad de cada uno de los grupos fue medida mediante la técnica del Press-o-Film ${ }^{\circledR}$, una técnica estandarizada -según el método C de la norma ASTM D4417-- aplicada para medir rugosidad de películas delgadas y que en este caso ha sido adaptada para proporcionar una medida aproximada de la rugosidad superficial de los agregados. La Figura 4 ilustra las cintas sensibles a presión suministradas por Testex y el sistema micrométrico utilizado para la determinación cuantitativa de rugosidad. La imagen en la Figura 5 muestra agregados de "rugosidad" visual de acuerdo a la clasificación por grupos.

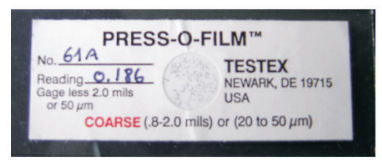

(a)

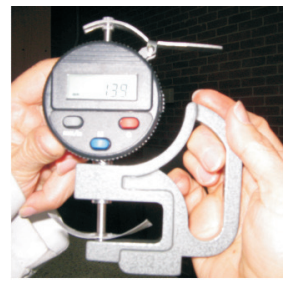

(b)
Figura 4. Dispositivo utilizado para la determinación de la rugosidad superficial. (a) Cinta calibrada sensible a la presión. (b) Micrómetro digital para medición de las cintas. 


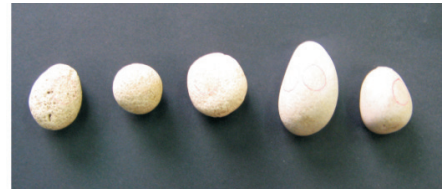

Figura 5. Muestra típica de los cinco grupos de agregados clasificados. La rugosidad visual disminuye de izquierda a derecha.

Además todas las muestras son examinadas bajo microscopio óptico binocular (Cole-Parmer). Las imágenes de textura superficial obtenidas mediante microscopia son capturadas a resoluciones de $4 X$ y $10 X$ utilizando la cámara CCD acoplada al microscopio. Las imágenes a color originales son posteriormente transformadas a imágenes en escala de grises y recortadas para formar imágenes de $640 \times 480$ píxeles. Estas imágenes son transformadas en imágenes en blanco y negro mediante la aplicación de un umbral (threshold) apropiado, dichas imágenes son utilizadas sin ninguna otra transformación o mejoramiento digital para la determinación de la dimensión fractal y la lacunaridad. Una muestra típica de imágenes utilizadas en este trabajo se muestra en la Figura 6. A menos que se indique lo contrario las imágenes utilizadas para todos los análisis son las correspondientes a una resolución de $4 \mathrm{X}$.

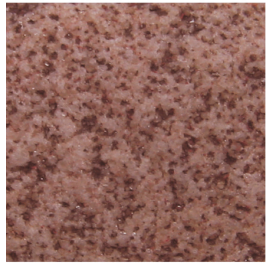

(a)

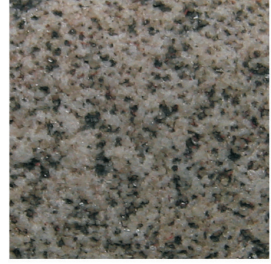

(b)

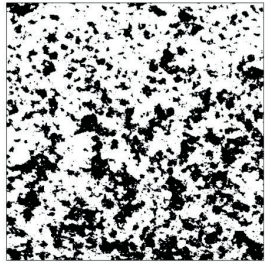

(c)
Figura 6. Muestra de imágenes de textura superficial. (a) imagen original (b) imagen en escala de grises (c) imagen en blanco y negro luego de realizar la transformación binaria.

\section{RESULTADOS Y DISCUSIÓN}

La gran mayoría de los perfiles de rugosidad son irregulares, y por lo tanto para propósitos de análisis teó- rico se consideran como la realización de un proceso aleatorio, que satisface condiciones de ergodicidad y normalidad. Basados en estas suposiciones, es posible analizar un perfil de rugosidad como una característica estadística. Como ya se mencionó arriba estas suposiciones no se satisfacen en la mayoría de los casos. Por tanto es necesario desarrollar nuevas metodologías para describir la rugosidad superficial. Las superficies reales son -en ciertas escalas de longitud--, parcialmente fractales, de tal forma que es natural aproximarlas y modelarlas aplicando modelos y características derivados de la geometría fractal. Dos de tales descriptores se analizan a continuación.

\section{A. Rugosidad superficial y dimensión fractal}

Desde el punto de vista clásico, es posible evaluar muchos parámetros de rugosidad basándose en el perfil de la superficie. Una descripción completa de tales parámetros se ha recopilado en [10]. Una característica no clásica aplicada por muchos investigadores para caracterizar la uniformidad (suavidad) de una superficie, es la dimensión fractal $D_{s}$. Para perfiles en dos dimensiones se tiene que $1<D_{s}<2$, donde 1 corresponde a una superficie lisa y 2 a una superficie altamente rugosa.

La dimensión fractal de los agregados estudiados se ha evaluado mediante técnicas de análisis de imagen, aplicando el software VisiQuest ${ }^{\circledR}$ de Accusoft, donde se aplica el algoritmo pml para la determinación de la dimensión fractal. La dimensión fractal así determinada se presenta en la Tabla 1

Tabla 1. Dimensión fractal estimada, aplicando técnicas de análisis de imágenes.

\begin{tabular}{|c|c|c|}
\hline Grupo & $\langle\mathbf{h}\rangle[\mu \mathbf{m}]$ & Dimensión fractal $D_{s}$ \\
\hline 1 & 142.0 & 1.615 \\
\hline 2 & 129.8 & 1.664 \\
\hline 3 & 116.8 & 1.688 \\
\hline 4 & 100.0 & 1.711 \\
\hline 5 & 50.14 & 1.750 \\
\hline
\end{tabular}


Los resultados en la Tabla 1 indican que el valor de la dimensión fractal aumenta a medida que el valor promedio de la altura de las asperezas disminuye. La Figura 7 ilustra de manera grafica este comportamiento

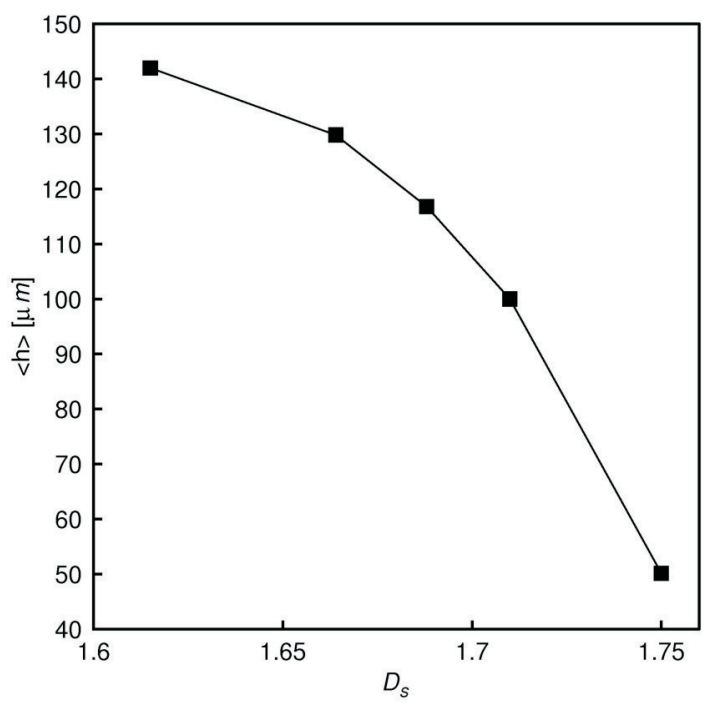

Figura 7. Variación de la rugosidad superficial promedio medida mediante la técnica de la cinta sensible a la presión (Press-oFilm ${ }^{\circledR}$ ) con la dimensión fractal $D_{s}$. Los resultados son el promedio de cinco observaciones diferentes

Los resultados en la Figura 7 están cualitativamente de acuerdo con los datos experimentales reportados por Macenaite et al. [11], quienes determinaron el comportamiento de la rugosidad promedio con la dimensión fractal para muestras de caucho tratadas con abrasivos de diverso tamaño de grano. La relación (correlación) existente entre $\langle\mathrm{h}\rangle$ y $D_{s^{\prime}}$--que se puede establecer de acuerdo con los datos en la Tabla 1--, puede en principio ser utilizada para determinar la rugosidad superficial promedio, usando la dimensión fractal como único parámetro, tal y como lo han sugerido Macenaite et al. Sin embargo, existen limitaciones serías con esta aproximación como se ilustra a continuación.

\section{B. Rugosidad superficial y lacunaridad}

Lacunaridad visual. Considérese la imagen que se muestra en la Figura 8 y que corresponde a un arreglo de diversos conjuntos de Cantor [4], reproducidos de [12]. Todos y cada uno de estos conjuntos poseen la misma dimensión fractal $D=1 / 2$, sin embargo difieren de una manera muy obvia para el observador. Los mostrados al fondo de la Figura 8 muestran agujeros (espaciamientos) más pequeños y se denominan de baja lacunaridad. Los que se encuentran en la parte superior poseen agujeros (espaciamientos) grandes y se denominan de alta lacunaridad. Esta observación pone de manifiesto un hecho genérico. Una geometría con una dimensión fractal $D$ dada puede representar un rango muy amplio de texturas, esto indica de manera irrefutable que la dimensión fractal por si sola no puede usarse para caracterizar una geometría y en particular la rugosidad superficial. Un ejemplo complementario aplicado al contexto que nos ocupa, esto es, rugosidad de superficies se ilustra en la Figura 9. Todas las imágenes en la Figura 9, poseen una dimensión fractal similar y aproximadamente igual a $D=1.91$, sin embargo como es obvio para el lector la textura de dichas superficies es totalmente diferente.

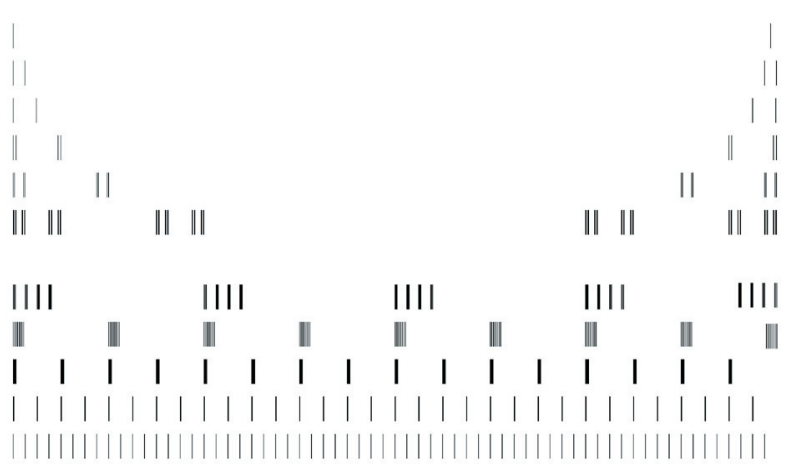

Figura 8. Serie de conjuntos de Cantor de igual dimensión fractal $D_{s}=1 / 2$, cuya lacunaridad cambia de muy baja en el fondo a muy alta en la parte superior. Adaptado de Ref. [12] 


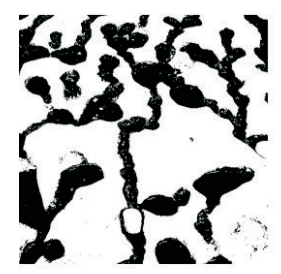

(a)

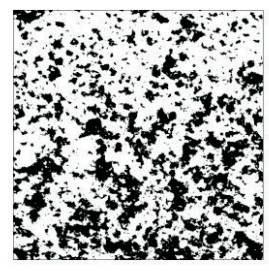

(b)

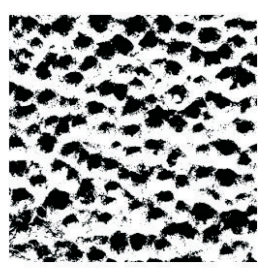

(C)
Figura 9. Variación de la lacunaridad en superficies rugosas para un valor constante de $D_{s}=1.91$. Lacunaridad (a) $\lambda=2.59$, (b) $\lambda=2.15$, (c) $\lambda=1.96$. Tanto el valor de $D_{s}$ como de $\lambda$ fueron determinados aplicando análisis de imagen aplicando Fraclac para ImageJ.

Físicamente, la lacunaridad puede entonces interpretarse como una medida de la distribución de los espacios de una geometría dada. Una definición precisa de lacunaridad sin embargo muestra que se trata de una medida de desviación de la geometría de un objeto --tal como un fractal-- a la invarianza translacional. Esto es, a una escala de longitud dada, que tan similares son diversas regiones de un objeto geométrico con respecto a otras. La lacunaridad puede por lo tanto considerarse como una medida de heterogeneidad o textura de un objeto -dependiente de la escala--, sea este fractal o no.

Existe una necesidad muy importante de medir cuantitativamente la diferencia en textura entre diversos materiales y la lacunaridad parece ofrecer una alternativa razonable. La lacunaridad de los agregados estudiados (ver Figura 5) se ha evaluado aplicando técnicas de análisis de imagen, mediante el aplicativo Fraclac de ImageJ, software desarrollado por el Instituto Nacional de Salud (USA), y de dominio publico bajo licencia GNU.

Tabla 2. Lacunaridad estimada, aplicando técnicas de análisis de imágenes.

\begin{tabular}{|c|c|c|}
\hline Grupo & $<\mathbf{h}>[\mu \mathbf{m}]$ & Lacunaridad \\
\hline 1 & 142.0 & 2.323 \\
\hline 2 & 129.8 & 2.224 \\
\hline 3 & 116.8 & 1.963 \\
\hline 4 & 100.0 & 1.656 \\
\hline 5 & 50.14 & 1.084 \\
\hline
\end{tabular}

Los resultados en la Tabla 2 indican que el valor de lacunaridad disminuye a medida que el valor promedio de la altura de las asperezas $\langle\mathrm{h}\rangle$ disminuye La Figura 10 ilustra dicho comportamiento. Recuérdese que la lacunaridad es una medida de la varianza de los espaciamientos de una geometría. En este contexto, si los espaciamientos de las asperezas sobre la superficie son uniformes sobre toda la superficie, entonces la varianza y por lo tanto el valor de lacunaridad, son bajos. Si el espaciamiento es grande y/o concentrado en ciertas áreas de la superficie, tanto la varianza de los espaciamientos y por tanto el valor de lacunaridad serán altas.

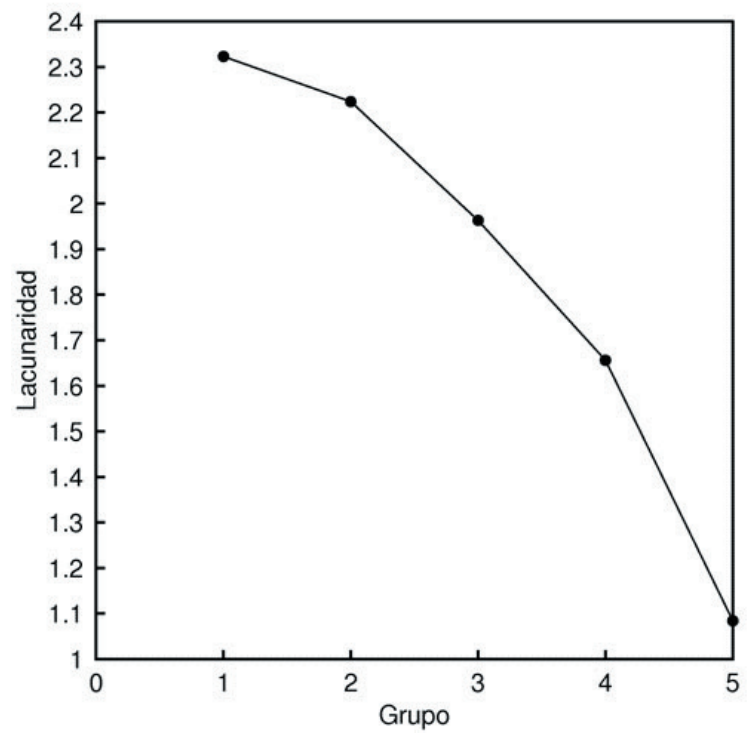

Figura 10. Variación de la lacunaridad determinada mediante técnicas de análisis de imagen de acuerdo a la clasificación visual por grupos. Los resultados son el promedio de cinco observaciones diferentes.

La Figura 11 ilustra el comportamiento de lacunaridad con la altura media de las asperezas, determinada aplicando la técnica de la cinta sensible a la presión (Press-o-Film ${ }^{\circledR}$ ). Nótese que el comportamiento de lacunaridad con $\langle h\rangle$, sigue un compartimiento perfectamente intuitivo. Esto es, la lacunaridad incrementa con el incremento de la altura media de las 
asperezas $\langle h\rangle$, esto hace de esta característica un parámetro muy útil para propósitos de análisis.

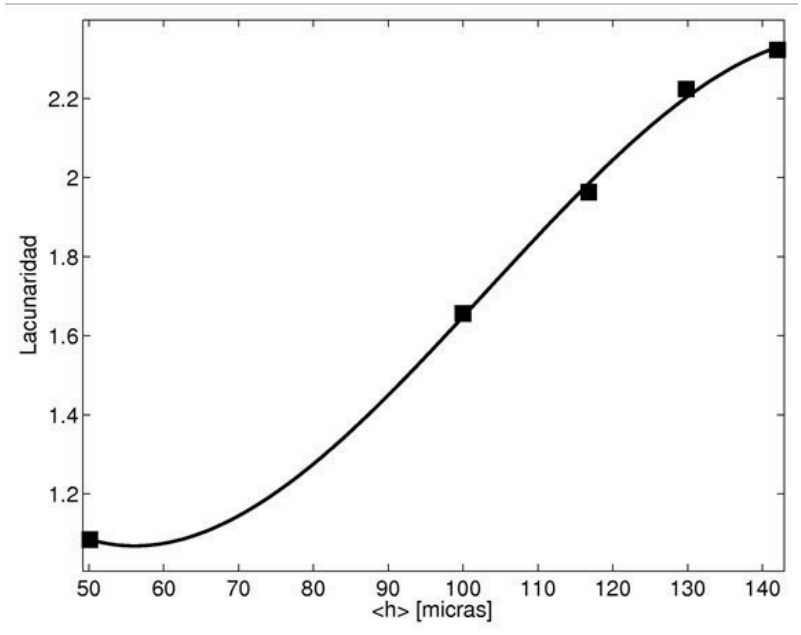

Figura 11. Relación entre la rugosidad superficial promedio y la lacunaridad. La línea continua representa una guía para la vista.

Debe mencionarse que procedimientos similares han sido aplicados para estudiar la textura y lacunaridad de diversas estructuras naturales de interés tales como agregados coloidales, micro-estructura de materiales porosos y compuestos, distribución de galaxias, distribución urbanística, imágenes médicas, etc. $[11,13,14,15,16]$

Muchos investigadores han usado la dimensión fractal como parámetro para caracterizar la forma como se "ve" una superficie. Sin embargo, es claro que la dimensión fractal por si sola no puede describir de manera completa la apariencia visual, dado que no puede cuantificar las características que describen el llenado del espacio. La dimensión fractal mide cuanto espacio está lleno. La lacunaridad complementa a la dimensión fractal midiendo como las geometrías Ilenan el espacio [17].

\section{CONCLUSIONES Y PERSPECTIVAS}

Aun cuando los métodos fractales están siendo aceptados como aproximaciones estándar en el análisis de patrones espaciales, dichos métodos son en la mayoría de los casos inadecuados para describir la textura de objetos reales en todo el rango de patrones. Las texturas reales pueden o no ser fractales; y cuando existe estructura fractal, esta solo existe en un rango limitado de ordenes de magnitud, por lo que patrones con la misma dimensión fractal pueden verse diferentes, i.e, tener diferentes texturas [4]. Esta observación es muy importante en el contexto de rugosidad superficial, dado que indica que la dimensión fractal por si sola no se puede utilizar como parámetro característico de rugosidad de una superficie dada, como se ha sugerido en algunas publicaciones.

En contraste, el análisis de lacunaridad, es un método más general [15]. Se puede aplicar a datos de cualquier dimensión, a patrones fractales, multi-fractales y no fractales. Este método permite determinar variaciones de la estructura espacial aún para patrones con dependencia espacial. La técnica es relativamente fácil de implementar y provee gráficos que son fáciles de interpretar. En opinión de los autores, está técnica puede ser aplicada exitosamente en el análisis cualitativo y cuantitativo de la rugosidad superficial tal y como se ilustra en los análisis efectuados en el presente estudio. Los resultados muestran que superficies donde los vacíos tienen diversos tamaños presentan valores altos de lacunaridad, mientras que las superficies cuyos tamaños de vacíos son uniformes presentan lacunaridades bajas. Por lo tanto una clasificación de superficies rugosas basada en lacunaridad está en concordancia perfecta con lo que podría ser una clasificación por inspección de las superficies y ofrece la posibilidad de cuantificar la 
noción un poco elusiva de "rugosidad". Esta aproximación sin embargo requiere de estudios aún más detallados para poder ser estandarizada.

\section{Agradecimientos}

Los autores expresan su agradecimiento al personal de los laboratorios de Ingeniería Civil de la Universidad Militar Nueva Granada (UMNG) por su colaboración en el desarrollo de las medidas experimentales. Este trabajo es financiado mediante el proyecto ING2005-05 de la UMNG. El trabajo de Lyda M. Pineda, es apoyado financieramente mediante el programa de Jóvenes Investigadores de la UMNG.

\section{REFERENCIAS}

[1] PERESSAKO, A. G. HOSODA, N. and PERSSON, B.N.J. Physical Review Letters 95, 124301 (2005).

[2] BERgERON, V. BERGER, C. and BETTERTON, M. D. Physical Review Letters 96, 098502 (2006).

[3] BLACKMORE, D. and ZHOU, J. G. SIAM Journal of Applied Mathematics 56, 1694-1719 (1996).

[4] MANDELBROT, M. The Fractal Geometry of Nature (Freeman, New york, 1982)

[5] MAJUMDAR, A. and TIEN, C. Wear 136, 313-327 (1990).

[6] CIAVAARELLA, M. et al., Proc. R. Soc. Lond. A 456, 387-405 (1999).

[7] PERSSON, B. N. J. Journal of Chemical Physics 115, 3840-3861 (2001).

[8] YAN, W. and KOMVOPOULOS, K. Journal of Applied Physics 84, 3617-3624 (1998).

[9] AUSLOOS, M. and BERMAN, D. H. Proc. R. Soc.
London, Ser. A 400, 331 (1985).

[10] GADELWALA, E. S. et al., Journal of Materials Processing Technology 123, 133-145 (2002).

[11] MACENAITE, L. PEKARSKAS, V. and ZUNDA, A. Materials Science 10, 268 (2004).

[12] BLUMENTFELD, R. and MANDELBROT, B. B. Physical Review E 56, 112 (1997).

[13] MUREIKA, J. R. and DYER, C. C. arXiv.orgqc/0505083 1, 1 (2005).

[14] GREENHILL et al. Use of Lacunarity index to characterize sub-urban areas for land use planning using IKONOS-2 multispectral imagery (2002).

[15] PLOTNICK, R. E. GARDNER, R. H. and O'NEILL, R. V. Landscape Ecology 8, 201-211 (1993).

[16] HUANG, Y.-H. and CHENG, S.-C. International Journal of Computer Applications in Technology 22, 34-41 (2005).

[17] TOLLE, C. R. et al., Physica D 179, 129-152 (2003). 